\title{
Mental health and burnout in primary and secondary school teachers in the remote mountain areas of Guangdong Province in the People's Republic of China
}

\author{
This article was published in the following Dove Press journal: \\ Neuropsychiatric Disease and Treatment \\ 17 January 2014 \\ Number of times this article has been viewed
}

\section{Lulu Zhang' \\ Jingping Zhao' \\ Huaqing $\mathrm{Xiao}^{3}$ \\ Hongbo Zheng ${ }^{2}$ \\ Yaonan $\mathrm{Xiao}^{3}$ \\ Miaoyang $\mathrm{Chen}^{3}$ \\ Dingling Chen ${ }^{3}$}

'Mental Health Institute of the Second Xiangya Hospital, Key Laboratory of Psychiatry and

Mental Health of Hunan Province, Central South University, Changsha, Hunan, ${ }^{2}$ Department of Psychiatry, Guangzhou Brain Hospital, Affiliated Hospital of Guangzhou Medical University, Guangzhou, Guangdong, ${ }^{3}$ Department of Psychiatry, Luoding Dagang Hospital, Luoding, Guangdong, People's Republic of China
Correspondence: Jingping Zhao 139 Middle Renming Road, 2nd Xiangya Hospital of Central South University, Changsha, Hunan 4I00I I, People's Republic of China

Email zhaojingpingcsu@।63.com
Background: A growing number of studies have shown that education is a work context in which professionals (teachers) seem likely to suffer from burnout that may be associated with low levels of mental health. Although there is a demonstrated need to improve the mental health and burnout levels among teachers, little is known about their mental health status, particularly with respect to graduating class teachers in remote mountain areas with undeveloped economies. The purpose of this study was to survey mental health and burnout among graduating class teachers in remote mountain areas and to examine the influence of moderating variables.

Methods: We conducted a multilevel analysis of 590 graduating class teachers from 42 primary and secondary schools in remote mountain areas of Guangdong province in the People's Republic of China. The outcome variable of self-reported mental health was measured by the Symptom Checklist-90 (SCL-90), and burnout was measured by the Chinese Maslach Burnout Inventory for primary and secondary school teachers.

Results: The status of both mental health and burnout among the respondents was significantly more troubling than the national norm used as a reference $(P<0.05$ or $P<0.01)$. Each factor in the SCL-90 had a significant correlation with burnout $(P<0.01)$. All factors of the SCL-90 were entered into the regression equation for each dimension of burnout $(P<0.01)$. The factor having the greatest impact on emotional exhaustion and depersonalization was anxiety (beta 0.187 and 0.178 , respectively). The factor having the greatest impact on reduced personal accomplishment and intellectual burnout was somatization (beta -0.214 and 0.185 , respectively).

Conclusion: The current outlook for the status of mental health and burnout among teachers in remote mountain areas of Guangdong is not good. The level of mental health among these teachers is lower than the national average, and the level of burnout is higher. Mental health status has obvious effects on burnout among these teachers.

Keywords: mental health, burnout, teacher, Symptom Checklist-90, Chinese Maslach Burnout Inventory

\section{Introduction}

The mental health of teachers is a basic component of the quality of education and affects both teachers' work achievement and the mental health of students. ${ }^{1}$ Burnout, or professional exhaustion syndrome, is characterized by feelings of physical and emotional exhaustion, depersonalization, and reduced personal accomplishment. ${ }^{2,3}$ Studies have shown that education is a work context in which professionals (teachers) seem to be more inclined to suffer from burnout, which may be associated with a low level of mental health. ${ }^{2,4-8}$ Among the reasons for premature retirement 
among German teachers, the most common (at 52\%) were psychiatric or psychosomatic diagnoses. A large part of these diagnoses consisted of depression, stress disorders, and exhaustion syndromes, all of which overlap with the established symptoms of so-called burnout syndrome. ${ }^{9}$ A primary and secondary students' mental health education research group in the People's Republic of China has indicated that approximately half of all teachers have psychological problems. ${ }^{10}$

In the last two decades, the People's Republic of China has deepened its basic education reforms through significant changes to school systems and teachers' evaluation systems. These changes have led to more pressure on teachers, who are, in turn, more likely to experience negative emotions and physical discomfort. ${ }^{11}$ The mental health of teachers has become an important part of public health. Burnout is not the same as being depressed or overworked. It is a subtle process in which an individual is gradually caught in a state of mental fatigue and is completely empty and drained of all energy. Maslach et al described burnout as a syndrome of emotional exhaustion, depersonalization, and reduced personal accomplishment. ${ }^{3}$ Burnout syndrome is a problem born of good intentions because it happens when individuals try to reach unrealistic goals and end up depleting their energy and losing touch with themselves and others. ${ }^{12}$ Given the specific characteristics of contemporary Chinese teachers, Fang et al designed the Chinese Primary and Secondary School Teachers Maslach Burnout Inventory (C-MBI) and established a national norm. ${ }^{13}$ The $\mathrm{C}-\mathrm{MBI}$ is an effective tool to evaluate burnout among primary and secondary school teachers in the People's Republic of China and has good reliability and validity. Although there are previous studies of the matter, evidence of the association between mental health and burnout among Chinese teachers is limited by the fact that little empirical research has been conducted that adopts a multilevel framework to assess the association of mental health levels of teachers in remote mountain areas with burnout.

Luoding city is located in western Guangdong and is one of 50 remote mountain regions in the province. According to the Guangdong statistical yearbook in 2012 , Luoding is not well developed economically; in 2011, the per capita annual income was 10,937 Chinese yuan, which is the lowest-ranking of the 121 counties in Guangdong. Mountain area teachers are at an increased risk of psychological problems because of low income, inconvenient transport, inaccessible information, and a difficult physical and cultural life. ${ }^{14}$ In this locality, more and more parents expect their children to escape poverty through attending school. However, there is a large gap between educational needs and the poor teaching environment, which increases the burden on teachers. Influenced by local traditional culture, female teachers are expected to take on more housework at home and perform the same tasks as males at work. They may face more pressure than male teachers. Burnout is a distinct work-related syndrome and is most likely to occur in jobs that require extensive care for others. ${ }^{15}$ Teaching is a career with a particularly high risk of burnout. ${ }^{2}$ Graduating class teachers in primary and secondary schools, particularly those faced with an excessive workload and high enrollment rate, may feel greater pressure. However, a serious lack of scientific knowledge hampers the efforts of education departments to prevent psychological problems and burnout among teachers. We selected members of this occupational group in a remote mountain area, who have often been neglected, as the respondents for our study.

This study focused on mental health and burnout levels among teachers in remote mountain areas with undeveloped economies. Our aim was to survey and analyze the relationship between mental health and burnout among graduating class teachers in Luoding, Guangdong, People's Republic of China.

\section{Subjects and methods Ethics}

The study protocol was reviewed and approved by the institutional review board of the Luoding Health Bureau. All participating schools deferred to the Luoding Health Bureau's institutional review board approval of the study protocol. We obtained written consent from all study participants.

\section{Study design and participation}

Using a stratified sampling method, our study surveyed primary and secondary school teachers in Luoding from November 2011 to April 2012. One primary school and one secondary school were randomly sampled in each of the 21 "towns" or districts that comprise Luoding, and 15 graduating class teachers in each sampled school were randomly selected for interview and questionnaire investigations. A total of 630 copies of the questionnaire were provided to 42 primary and secondary schools, and 590 questionnaires were collected, resulting in an effective rate of $93.65 \%$. Among the 590 valid questionnaires, there were 204 males with an average age of $34.46 \pm 8.11$ years and 386 females with 
an average age of $34.01 \pm 7.67$ years. There was no significant difference in age between sexes in the respondents.

\section{Study instruments}

The outcome variable of self-reported mental health was measured by the Symptom Checklist-90 (SCL-90). The SCL-90 has been translated into Chinese and provides an empirical test of validity. ${ }^{10,16}$ The SCL-90 includes nine symptom factors, ie, somatization, obsessive-compulsive, interpersonal sensitivity, depression, anxiety, hostility, phobia, paranoia, and psychoticism. A factor score $\geq 2$ points indicates "mild symptoms", and $\geq 3$ points indicates "at least moderate symptoms".

In addition to the SCL-90, the questionnaire incorporated the C-MBI to assess teacher burnout. This scale was designed by Fang et al, who added the dimension of intellectual burnout on the basis of the Maslach Burnout Inventory-Educator Survey. ${ }^{13}$ Thus, the C-MBI comprises four dimensions, ie, emotional exhaustion, depersonalization, reduced personal accomplishment, and intellectual burnout. The first three follow the three aspects of the Maslach Burnout Inventory-Educator Survey. The last dimension is intended for the specific characteristics of Chinese primary and secondary school teachers and is presented as follows: teachers do not adapt to rapid social changes and knowledge updating and have difficulty coping with students' problems and poor learning abilities; as a result, they experience a sense of intellectual exhaustion. ${ }^{13}$ The study was conducted by trained psychiatrists to ensure its quality.

\section{Statistical analysis}

A statistical analysis of the data was conducted using Statistical Package for the Social Sciences version 17.0 software (SPSS Inc., Chicago, IL, USA). A two-sided test of significance was used, and the level of statistical significance was $P<0.05$. All data were expressed as the mean \pm standard deviation. A Student's $t$-test was used to compare the differences between the two groups. The national norms for the SCL-90 and C-MBI were used as a reference for the national average level of mental health and burnout, respectively. A partial correlation analysis with sex as the control variable was conducted to examine the correlation between mental health and burnout. Stepwise multiple regression was used to analyze the impact of burnout on mental health, in which four dimensions of burnout were the dependent variables and nine factors of the SCL-90 were the independent variables (criteria being probability-of-F-to-enter $<0.05$, probabilityof-F-to-remove $>0.10$ ).

\section{Results \\ Mental health outcomes}

As shown in Table 1, the total score for the SCL-90 among the respondents was significantly higher than that of the national norm $(P<0.01)$, and each factor score increased significantly $(P<0.01)$, with the exception of interpersonal sensitivity and phobia. The mean factor score for obsessive-compulsive characteristics was more than two points. The data suggest that the overall mental health of respondents was at a low level, perhaps with some psychological problems and mild obsessive-compulsive symptoms.

Further analysis (Table 2) showed that the total score and each factor score on the SCL-90 among female teachers were all significantly higher than those of the male teachers $(P<0.01)$. This result suggests that mental health in female teachers is worse than that of male teachers. Moreover, there were no significant differences in either total score or in each factor score among age groups, educational levels, professional titles, or seniority.

\section{Burnout outcomes}

As shown in Table 3, the respondents scored significantly higher on both emotional exhaustion and intellectual burnout and significantly lower on reduced personal accomplishment than the reference of the national norm $(P<0.05$ or $P<0.01)$. This result suggests that the respondents have a certain degree of burnout, such as self-experience of emotional exhaustion, reduced personal accomplishment, and intellectual burnout.

Further analysis (Table 4) showed that the factor scores for emotional exhaustion, depersonalization, and intellectual

Table I Comparison of scores on the SCL-90 of respondents with the national norm

\begin{tabular}{lccl}
\hline Items & $\begin{array}{l}\text { Respondents } \\
(\mathbf{n}=\mathbf{5 9 0})\end{array}$ & $\begin{array}{l}\text { National norm } \\
(\mathbf{n}=\mathbf{I}, \mathbf{3 8 8})\end{array}$ & $\boldsymbol{t}$ \\
\hline Factor & & & \\
$\quad$ Somatization & $1.87 \pm 0.54$ & $1.37 \pm 0.48$ & $19.46^{*}$ \\
$\begin{array}{l}\text { Score } \\
\text { Obsessive-compulsive }\end{array}$ & $2.03 \pm 0.5 \mathrm{I}$ & $1.62 \pm 0.58$ & $15.69^{*}$ \\
Interpersonal sensitivity & $1.70 \pm 0.49$ & $1.65 \pm 0.6 \mathrm{I}$ & 1.92 \\
Depression & $1.66 \pm 0.48$ & $1.50 \pm 0.59$ & $6.32^{*}$ \\
Anxiety & $1.54 \pm 0.50$ & $1.39 \pm 0.43$ & $6.36^{*}$ \\
Hostility & $1.59 \pm 0.54$ & $1.46 \pm 0.55$ & $4.87^{*}$ \\
Phobia & $1.27 \pm 0.51$ & $1.23 \pm 0.4 \mathrm{I}$ & 1.69 \\
Paranoia & $1.54 \pm 0.48$ & $1.43 \pm 0.57$ & $4.40^{*}$ \\
$\quad$ Psychoticism & $1.40 \pm 0.43$ & $1.29 \pm 0.42$ & $5.24^{*}$ \\
Total score & $168.73 \pm 36.19$ & $129.96 \pm 38.76$ & $21.33^{*}$ \\
\hline
\end{tabular}

Notes: All data are shown as the mean \pm standard deviation. $* P<0.0$. Abbreviation: SCL-90, Symptom Checklist-90. 
Table 2 Comparison of scores on the SCL-90 between sexes

\begin{tabular}{lccl}
\hline Items & $\begin{array}{l}\text { Female } \\
(\mathbf{n}=\mathbf{3 8 6})\end{array}$ & $\begin{array}{l}\text { Male } \\
(\mathbf{n}=\mathbf{2 0 4})\end{array}$ & $\begin{array}{l}\text { Student's } \\
\text { t-test }\end{array}$ \\
\hline Factor & & & \\
$\quad$ Somatization & $1.97 \pm 0.56$ & $1.66 \pm 0.45$ & $7.34^{*}$ \\
$\begin{array}{l}\text { Score } \\
\text { Obsessive-compulsive }\end{array}$ & $2.14 \pm 0.52$ & $1.85 \pm 0.43$ & $7.26^{*}$ \\
Interpersonal sensitivity & $1.77 \pm 0.51$ & $1.58 \pm 0.43$ & $4.89^{*}$ \\
Depression & $1.75 \pm 0.49$ & $1.50 \pm 0.40$ & $6.69^{*}$ \\
Anxiety & $1.64 \pm 0.51$ & $1.34 \pm 0.40$ & $7.66^{*}$ \\
Hostility & $1.68 \pm 0.56$ & $1.42 \pm 0.45$ & $6.18^{*}$ \\
Phobia & $1.37 \pm 0.53$ & $1.08 \pm 0.39$ & $7.36^{*}$ \\
Paranoia & $1.64 \pm 0.49$ & $1.38 \pm 0.42$ & $6.76^{*}$ \\
$\quad$ Psychoticism & $1.47 \pm 0.45$ & $1.27 \pm 0.35$ & $6.17^{*}$ \\
Total score & $176.81 \pm 37.16$ & $153.46 \pm 28.71$ & $8.46^{*}$ \\
\hline
\end{tabular}

Notes: All data are shown as the mean \pm standard deviation. $* P<0.01$.

Abbreviation: SCL-90, Symptom Checklist-90.

burnout were significantly higher among female teachers than in male teachers, whereas the factor score for reduced personal accomplishment was reversed $(P<0.01)$. The data suggest that burnout is more severe in female teachers than in male teachers. There were no significant differences in any dimension score among age groups, educational levels, professional titles, or seniority.

\section{Correlation between mental health and burnout}

As shown in Table 5, each factor on the SCL-90 had a significantly positive correlation with emotional exhaustion, depersonalization, and intellectual burnout, and had a significantly negative correlation with reduced personal accomplishment $(P<0.01)$. This result suggests that there may be a strong relationship between mental health and burnout.

\section{Burnout impact of mental health}

As shown in Table 6, all factors of the SCL-90 were entered into the regression equation for each dimension of

Table 3 Comparison of scores on the C-MBI of respondents with those of the national norm

\begin{tabular}{llll}
\hline Dimensions & $\begin{array}{l}\text { Respondents } \\
(\mathbf{n = 5 9 0 )}\end{array}$ & $\begin{array}{l}\text { National norm } \\
(\mathbf{n}=679)\end{array}$ & $\boldsymbol{t}$ \\
\hline EE & $25.90 \pm 4.76$ & $22.48 \pm 8.49$ & $9.00^{* *}$ \\
DP & $13.33 \pm 4.45$ & $12.92 \pm 4.59$ & 1.61 \\
PA & $18.61 \pm 2.42$ & $19.19 \pm 4.55$ & $-2.89^{* *}$ \\
IB & $8.59 \pm 4.17$ & $8.10 \pm 2.64$ & $2.46^{*}$ \\
\hline
\end{tabular}

Notes: All data are shown as the mean \pm standard deviation. ${ }^{*} P<0.05 ; * * P<0.01$. Abbreviations: EE, emotional exhaustion; DP, depersonalization; PA, reduced personal accomplishment; IB, intellectual burnout; C-MBI, Chinese Primary and Secondary School Teachers Maslach Burnout Inventory.
Table 4 Comparison of scores on the C-MBI between sexes

\begin{tabular}{llll}
\hline Dimensions & $\begin{array}{l}\text { Female } \\
(\mathbf{n}=\mathbf{3 8 6})\end{array}$ & $\begin{array}{l}\text { Male } \\
(\mathbf{n}=\mathbf{2 0 4})\end{array}$ & t-test \\
\hline EE & $26.95 \pm 4.92$ & $23.91 \pm 3.66$ & $8.49 *$ \\
DP & $14.30 \pm 4.60$ & $11.48 \pm 3.47$ & $7.70 *$ \\
PA & $18.06 \pm 2.42$ & $19.65 \pm 2.05$ & $-8.39 *$ \\
IB & $9.55 \pm 4.27$ & $6.78 \pm 3.30$ & $8.75 *$ \\
\hline
\end{tabular}

Notes: All data are shown as the mean \pm standard deviation. $* P<0.01$.

Abbreviations: $\mathrm{EE}$, emotional exhaustion; DP, depersonalization; PA, reduced personal accomplishment; IB, intellectual burnout; C-MBI, Chinese Primary and Secondary School Teachers Maslach Burnout Inventory.

burnout $(P<0.01)$. The factor having the greatest impact on emotional exhaustion and depersonalization was anxiety (beta $=0.187$ and 0.178 , respectively), and the factor having the greatest impact on reduced personal accomplishment and intellectual burnout was somatization (beta $=-0.214$ and 0.185 , respectively). These data suggest that mental health status had obvious effects on burnout among the respondents. The best predictors of burnout may be anxiety and somatization.

\section{Discussion}

Our study found that the mental health status of the respondents is troubling. In particular, the differences in somatization from the national norm were most obvious. This finding indicates that the respondents relieve psychological pressure through individual somatic symptoms initiated to attract the attention of others instead of developing positive methods of coping. In the long term, this situation may lead to psychosomatic illness and may affect the respondents' teaching. Additionally, the respondents had mild obsessive-compulsive symptoms, which may be related to teachers' professional characteristics. The teaching profession requires teachers to have exemplary behavior and strict scholarship, which can easily lead to perfectionism and forced personality tendencies.

Table 5 Partial correlation between mental health and burnout

\begin{tabular}{lllll}
\hline Factors & EE & DP & PA & IB \\
\hline Somatization & $0.743^{*}$ & $0.744^{*}$ & $-0.745^{*}$ & $0.752^{*}$ \\
Obsessive-compulsive & $0.796^{*}$ & $0.794^{*}$ & $-0.780^{*}$ & $0.804^{*}$ \\
Interpersonal sensitivity & $0.767^{*}$ & $0.768^{*}$ & $-0.744^{*}$ & $0.782^{*}$ \\
Depression & $0.790^{*}$ & $0.785^{*}$ & $-0.777^{*}$ & $0.800^{*}$ \\
Anxiety & $0.859^{*}$ & $0.857^{*}$ & $-0.834^{*}$ & $0.86 I^{*}$ \\
Hostility & $0.70 I^{*}$ & $0.702^{*}$ & $-0.685^{*}$ & $0.710^{*}$ \\
Phobia & $0.698^{*}$ & $0.705^{*}$ & $-0.675^{*}$ & $0.710^{*}$ \\
Paranoia & $0.75 I^{*}$ & $0.754^{*}$ & $-0.740^{*}$ & $0.762^{*}$ \\
Psychoticism & $0.752^{*}$ & $0.754^{*}$ & $-0.74 I^{*}$ & $0.767^{*}$ \\
\hline
\end{tabular}

Notes: $\mathrm{n}=590$; sex as control variable; $* P<0.01$.

Abbreviations: EE, emotional exhaustion; DP, depersonalization; PA, reduced personal accomplishment; IB, intellectual burnout. 
Table 6 Regression analysis of impact of mental health on burnout

\begin{tabular}{|c|c|c|c|c|c|c|c|}
\hline Dimensions & Factors & $R^{2}$ & $R^{2}$ & B & SE & Beta & $\begin{array}{l}\text { Student's } \\
\text { t-test }\end{array}$ \\
\hline \multirow[t]{10}{*}{ EE } & & 0.964 & 0.928 & & & & \\
\hline & Anxiety & & & 1.784 & 0.214 & 0.187 & $8.342^{*}$ \\
\hline & Obsessive-compulsive & & & $1.46 \mathrm{I}$ & 0.175 & 0.156 & $8.359 *$ \\
\hline & Interpersonal sensitivity & & & 1.243 & 0.174 & 0.128 & $7.134 *$ \\
\hline & Somatization & & & 1.520 & 0.148 & 0.174 & $10.270 *$ \\
\hline & Psychoticism & & & 1.383 & 0.194 & 0.125 & $7.130 *$ \\
\hline & Depression & & & 1.456 & 0.189 & 0.146 & $7.688^{*}$ \\
\hline & Hostility & & & 0.821 & 0.141 & 0.093 & $5.823^{*}$ \\
\hline & Phobia & & & 0.762 & 0.151 & 0.081 & $5.06 I^{*}$ \\
\hline & Paranoia & & & 0.826 & 0.180 & 0.084 & $4.590 *$ \\
\hline \multirow[t]{10}{*}{ DP } & & 0.964 & 0.929 & & & & \\
\hline & Anxiety & & & 1.596 & 0.200 & 0.178 & $7.990 *$ \\
\hline & Obsessive-compulsive & & & 1.340 & 0.163 & 0.153 & $8.209 *$ \\
\hline & Interpersonal sensitivity & & & 1.230 & 0.163 & 0.136 & $7.556^{*}$ \\
\hline & Somatization & & & 1.445 & 0.138 & 0.176 & $10.453^{*}$ \\
\hline & Psychoticism & & & 1.324 & 0.181 & 0.127 & $7.311 *$ \\
\hline & Hostility & & & 0.771 & 0.132 & 0.093 & $5.855^{*}$ \\
\hline & Depression & & & 1.245 & 0.177 & 0.133 & $7.032 *$ \\
\hline & Phobia & & & 0.831 & 0.141 & 0.095 & $5.907^{*}$ \\
\hline & Paranoia & & & 0.796 & 0.168 & 0.086 & $4.737^{*}$ \\
\hline \multirow[t]{10}{*}{ PA } & & 0.948 & 0.899 & & & & \\
\hline & Anxiety & & & -0.708 & 0.129 & -0.145 & $-5.475^{*}$ \\
\hline & Obsessive-compulsive & & & -0.698 & 0.106 & -0.146 & $-6.598 *$ \\
\hline & Paranoia & & & -0.497 & 0.109 & -0.099 & $-4.570 *$ \\
\hline & Somatization & & & -0.953 & 0.090 & -0.214 & $-10.638^{*}$ \\
\hline & Depression & & & -0.830 & 0.115 & -0.163 & $-7.238 *$ \\
\hline & Psychoticism & & & -0.773 & 0.117 & -0.137 & $-6.588 *$ \\
\hline & Interpersonal sensitivity & & & -0.520 & 0.105 & -0.105 & $-4.927^{*}$ \\
\hline & Hostility & & & -0.389 & 0.085 & -0.087 & $-4.566 *$ \\
\hline & Phobia & & & -0.296 & 0.091 & -0.062 & $-3.252^{*}$ \\
\hline \multirow[t]{10}{*}{ IB } & & 0.975 & 0.950 & & & & \\
\hline & Anxiety & & & 1.268 & 0.158 & 0.151 & $8.052^{*}$ \\
\hline & Obsessive-compulsive & & & 1.256 & 0.129 & 0.153 & $9.756 *$ \\
\hline & Interpersonal sensitivity & & & 1.197 & 0.128 & 0.141 & $9.324 *$ \\
\hline & Psychoticism & & & 1.392 & 0.143 & 0.143 & $9.748^{*}$ \\
\hline & Somatization & & & 1.422 & 0.109 & 0.185 & 13.043* \\
\hline & Depression & & & 1.353 & 0.140 & 0.154 & $9.694^{*}$ \\
\hline & Hostility & & & 0.729 & 0.104 & 0.094 & $7.024^{*}$ \\
\hline & Phobia & & & 0.745 & 0.111 & 0.091 & $6.713^{*}$ \\
\hline & Paranoia & & & 0.706 & 0.132 & $0.08 I$ & $5.330 *$ \\
\hline
\end{tabular}

Notes: $\mathrm{n}=590$; stepwise multiple regression was used with four dimensions of burnout as dependent variables and nine factors of SCL-90 as independent variables. Criteria: probability-of-F-to-enter $<0.05$ and probability-of-F-to-remove $>0.10 ; * P<0.01$.

Abbreviations: EE, emotional exhaustion; DP, depersonalization; PA, reduced personal accomplishment; IB, intellectual burnout; SCL-90, Symptom Checklist-90.

Our findings are consistent with several previous national studies. ${ }^{14,17,18}$ Luo et al reported that the mental health status of 182 teachers in remote mountain areas of Shanxi Province was significantly lower than the national norm. ${ }^{14}$ Liu et al and Zhang et al reported that rural middle school teachers had a low level of mental health. ${ }^{17,18}$ Studies abroad have also demonstrated that the mental health level of teachers has given rise to significant social concern and increased numbers of psychological interventions. ${ }^{1,19}$ Claro et al found that $28.6 \%$ of 120 basic suburban schoolteachers in Chile had possible emotional problems. ${ }^{1}$ Lodolo D'Oria et al found that the risk of teachers developing psychiatric disorders was 2, 2.5, and 3 times higher than that of clerks, health care professionals, and blue collar workers, respectively. ${ }^{19}$ Our results may relate to current social factors and local characteristics. Luoding is a remote mountain area with an underdeveloped economy. There are a number of objective factors that widen the gap between the strong sense of a valued professional role and 
actual social status, such as low wages, inconvenient transport, a poor teaching environment and facilities, limited staffing, and a lack of mental health education. Moreover, the respondents in our study were graduating class teachers who faced greater pressure and a difficult workload. All of these factors may have aggravated their psychological problems.

Regarding burnout, our study showed that respondents exhibited some degree of burnout and mainly displayed emotional exhaustion, intellectual burnout, and reduced personal accomplishment. Among these self-reported experiences, emotional exhaustion was the most significant, followed by reduced personal accomplishment. These results coincide with the results of burnout models. ${ }^{20-25}$ Teachers are faced with an excessive emotional work burden, and their emotional resources are gradually depleted, ultimately resulting in emotional exhaustion. Furthermore, teachers find that there is a large gap between idealized expectations and reality, which results in a reduced sense of personal accomplishment. Other studies have reported that teachers are particularly prone to burnout. ${ }^{2,4}$ We did not find obvious depersonalization among our respondents, which is consistent with the results of the SCL-90, without significantly higher interpersonal sensitivity. This finding suggests that the respondents do not adopt a defensive coping style to reduce emotional input, but are inclined to adopt a somatization coping style to divert their experiences of negative emotion and emotional exhaustion. In addition, the respondents had significant levels of intellectual burnout, perhaps because teachers in remote mountain areas lack advanced studies and training.

With respect to sex differences in teachers' mental health and burnout, we found that psychological and burnout problems were more serious among female teachers than among male teachers, which is consistent with previous studies. ${ }^{18,26-28}$ Possible explanations for this phenomenon are related to the physical and psychological traits of females and to the local cultural environment. ${ }^{14}$ In Luoding, it is generally believed that females should take on more housework in families and should perform the same tasks as males at work. Moreover, female teachers are traditionally expected to be kinder, more patient, and more careful than males. Therefore, female teachers are under greater pressure, and they often show more internal conflict and maladaptive behavior, which may lead to physical discomfort, anxiety, depression, and burnout. We did not find significant differences in mental health or burnout among the different age groups, educational levels, professional titles, or seniority. This finding may be related to the overall low level of teachers' qualifications in remote mountain areas.

Furthermore, we found a significant relationship between mental health and burnout; respondents who scored high on negative affect also scored high on burnout. The level of mental health was a common predictor of burnout (emotional exhaustion accounted for $92.8 \%$ of the burnout cases, depersonalization for $92.9 \%$, reduced personal accomplishment for $89.9 \%$, and intellectual burnout for $95.0 \%$ ). The best predictor of emotional exhaustion and depersonalization was anxiety, and the best predictor of reduced personal accomplishment and intellectual burnout was somatization. This finding suggests that the symptom-related factors of the SCL-90, particularly anxiety and somatization, have important effects on burnout. Thus, regular assessment of teachers' mental health and early intervention are important to reduce burnout, which can be overcome when the cause is identified. On the one hand, it is necessary to undertake certain measures to improve the mental health of teachers, such as developing better teaching environments and living standards, establishing reasonable education policies, and strengthening social support systems. On the other hand, government departments should reinforce mental health and burnout education for teachers to help them establish rational cognition and realistic catharsis and manage psychological exhaustion. ${ }^{29,30}$ Moreover, schools in remote areas should attach particular importance to preventing intellectual burnout among teachers by providing professional training and career development planning.

Our study has certain limitations. The first is related to the assessment tools. Our outcome variables were selfreported. Given that depressed individuals are less likely to rate personal accomplishment in a positive light, this would lead to a common method bias. Another limitation may be the sampling of schools. Despite intense efforts to involve all schools, those that did not agree to participate may be less concerned about and supportive of their teachers, which may result in underestimated associations between mental health and burnout. Moreover, respondents might have concealed their true psychological status because of the reputation and authority of their schools. Additionally, stressors were not included in our survey content. Finally, there may be a difference between urban and rural teachers. Although developing this issue was beyond the scope of our paper, we consider this a potentially important issue for further exploration.

Our study also has some strengths. To our knowledge, this is the first study of mental health and burnout among primary and secondary school teachers in Guangdong Province. 
In particular, we focused on teachers in remote mountain areas, who have often been neglected. We measured each dimension of burnout and tested the influence of mental health on burnout. By understanding the present status of psychological problems and burnout among teachers, we can provide organizations with a scientific basis to improve the mental health levels of teachers and to find solutions. Our findings suggest that the current outlook for the status of mental health and burnout among graduating class teachers in remote mountain areas is not good. Promotion of mental health may be important to prevent burnout.

\section{Conclusion}

The current level of impaired mental health among graduating class teachers in remote mountain areas of Guangdong is lower than the national average level, and the level of burnout in these teachers is higher than the national average. Mental health status has obvious effects on burnout among these teachers. The troubling status of mental health and burnout among the teachers in this region warrants close attention, and the data presented here should enable us to highlight key areas that may be improved to enhance mental health and prevent burnout.

\section{Acknowledgments}

This study was supported by the Science and Technology Planning Project of Guangdong Province, China (2010B031600322). We thank the focal persons of the participating schools for their interest in the study and for arranging interviews and questionnaire investigations. We thank the teachers for taking time out of their busy schedules to participate in this study.

\section{Disclosure}

The authors report no conflicts of interest in this work. The findings and conclusions in this report are those of the authors and do not necessarily represent the official position of the Luoding Health Bureau.

\section{Author contributions}

LZ, JZ, HX, HZ, YX, and MC conceived and designed the experiments. LZ, JZ, HX, and HZ performed the experiments. LZ, JZ, and HX analyzed the data. LZ, JZ, HX, HZ, YX, MC, and DC contributed reagents, materials, and analytical tools. LZ and JZ wrote the paper. All authors contributed toward data analysis, drafting and revising the manuscript.

\section{References}

1. Claro S, Bedregal P. Mental health status of teachers in 12 schools of Puente Alto, Santiago, Chile. Rev Med Chil. 2003;131(2):159-167.
2. Chennoufi L, Ellouze F, Cherif W, Mersni M, M'rad MF. [Stress and burnout among Tunisian teachers]. Encephale. 2012;38(6):480-487. French.

3. Maslach C, Schaufeli W, Leiter M. Job burnout. Annu Rev Psychol. 2001;52(30):397-422.

4. Unterbrink T, Hack A, Pfeifer R, et al. Burnout and effort-rewardimbalance in a sample of 949 German teachers. Int Arch Occup Environ Health. 2007;80(5):433-441.

5. Zhong J, You J, Gan Y, et al. Job stress, burnout, depression symptoms, and physical health among Chinese university teachers. Psychol Rep. 2009;105(3 Pt 2):1248-1254.

6. David IC, Quintao S. [Burnout in teachers: its relationship with personality, coping strategies and life satisfaction]. Acta Med Port. 2012;25(3):145-155. Portuguese.

7. Kokkinos CM. Job stressors, personality and burnout in primary school teachers. Br J Educ Psychol. 2007;77 Pt 1:229-243.

8. Lodolo D'Oria V, Bulgarini d'Elci G, Bonomi P, et al. [Are teachers at risk for psychiatric disorders? Stereotypes, physiology and perspectives of a job prevalently done by women]. Med Lav. 2009;100(3):211-227. Italian.

9. Bauer J, Stamm A, Virnich K, et al. Correlation between burnout syndrome and psychological and psychosomatic symptoms among teachers. Int Arch Occup Environ Health. 2006;79(3):199-204.

10. Shande J. An analysis on SCL-90 of teachers of primary school in China. Chinese Mental Health Journal. 1993;7(2):78-79.

11. Zhu S, Dong LP, Du RH. [Investigation on occupational burnout and mental health of primary and secondary school teachers]. Chinese Journal of Industrial Hygiene and Occupational Diseases. 2009;27(3):165-166. Chinese.

12. Espeland KE. Overcoming burnout: how to revitalize your career. J Contin Educ Nurs. 2006;37(4):178-184.

13. Fang W, Yan X. Job burnout among elementary and high school teachers: characteristics and relationship with social support. Xin $\mathrm{Li}$ Хие Bao. 2004;36(5):568-574.

14. Luo Xiao-lan. An investigation on the mental health of teachers in remote mountain areas. Chinese Journal of Clinical Rehabilitation. 2006;10(6):165-167.

15. Lopes Cardozo B, Gotway Crawford C, Eriksson C, et al. Psychological distress, depression, anxiety, and burnout among international humanitarian aid workers: a longitudinal study. PLoS One. 2012;7(9): e44948.

16. Wang Wei. Qualitative and quantitative analysis on SCL-90 of minority undergraduates. China Journal of Health Psychology. 2012;20(3): 435-437.

17. Liu Yingli. Investigating the status of mental health of junior school teachers in underdevelopment areas. China Journal of Health Psychology. 2008;16(2):221-223.

18. Zhang Yongzhong, Deng Xianglan, Li Wenhu. Investigation of mental health state of junior high school teachers in underdevelopment area in country. China Journal of Health Psychology. 2006;14(1): $83-85$

19. Lodolo D’Oria V, Pecori Giraldi F, Della Torre M, et al. [Is there any correlation between psychiatric disease and the teaching profession?] Med Lav. 2004;95(5):339-353. Italian.

20. Leiter MP, Maslach C. The impact of interpersonal environment of burnout and organizational commitment. J Organ Behav. 1988;9(4): 297-308.

21. Golembiewski RT, Munzenrider RF, Stevenson JG. Phases of Burnout: Developments in Concepts and Applications. New York, NY, USA Praeger; 1988. Available from: http://www.getcited.org/pub/102638206. Accessed December 15, 2013.

22. Lee RT, Ashforth BE. A further examination of managerial burnout: toward an integrated model. J Organ Behav. 1993;14(1):3-20.

23. Lee RT, Ashforth BE. A longitudinal study of burnout among supervisors and managers: comparisons between the Leiter and Maslach (1988) and Golembiewski et al (1986) models. Organ Behav Hum Decis Process. 1993;54(3):369-398. 
24. Taris TW, Le Blanc PM, Schaufeli WB, Schreurs PJG. Are there causal relationships between the dimensions of the Maslach Burnout Inventory? A review and two longitudinal tests. Work Stress. 2005;19(3):238-255.

25. Parker PD, Salmela-Aro K. Developmental processes in school burnout: a comparison of major developmental models. Learn Individ Differ. 2011;21(2):244-248.

26. Lei W. Mental health of college teachers and its relationship with achievement motive. Journal of Clinical Rehabilitative Tissue Engineering Research. 2007;11(39):7834-7837.

27. Jingzhi W, Mingxi Z. A study on mental health of kindergarten, primary and middle school teachers. Chinese Journal of Clinical Psychology. 2004;12(3):306-308.
28. Nagai M, Tsuchiya KJ, Toulopoulou T, Takei N. Poor mental health associated with job dissatisfaction among school teachers in Japan. J Occup Health. 2007;49(6):515-522.

29. Jianqin L. Study of mental health level of primary and secondary school teachers in Xinxiang. China Journal of Health Psychology. 2004;12(4): 292-293.

30. Wei D, Bing F, Xiaoxia S. Mental health of teachers and the relationship between mental health and achievement motivation. Chinese Journal of Clinical Psychology. 2006;14(1):66-67.

\section{Publish your work in this journal}

Neuropsychiatric Disease and Treatment is an international, peerreviewed journal of clinical therapeutics and pharmacology focusing on concise rapid reporting of clinical or pre-clinical studies on a range of neuropsychiatric and neurological disorders. This journal is indexed on PubMed Central, the 'PsycINFO' database and CAS.
The manuscript management system is completely online and includes a very quick and fair peer-review system, which is all easy to use. Visit http://www.dovepress.com/testimonials.php to read real quotes from published authors.

Submit your manuscript here: http://www.dovepress.com/neuropsychiatric-disease-and-treatment-journal 